\title{
Problem Solving Strategy and Diagnostic Performance at Three Expertise Levels: Does the Problem Solving Approach Matter? \\ Fadi M Munshi' ${ }^{1 *}$, Badr M AlJarallah² and Peter H Harasym ${ }^{3}$
}

${ }^{1}$ Department of Medical Education and Educational Technology, Faculty of Medicine, King Fahad Medical City at King Saud Bin Abdulaziz University for Health Sciences, Riyadh, Saudi Arabia

${ }^{2}$ Department of Medicine, Qassim University, Qassim, Saudi Arabia

${ }^{3}$ Department of Community Health Sciences, Faculty of Medicine, University of Calgary, Calgary, Canada

\section{Abstract}

Objective: Saudi Arabia has expanded dramatically in the number of medical schools during the last 10 years. The objective of this study was to explore the knowledge structure, clinical problem solving approach, and diagnostic performance at three levels of expertise in the medical field.

Methods: This study involved twenty three participants. Fifth year medical students, internal medicine residents, and practicing internists were the participating medical staff representing novice, intermediate, and expert levels, consecutively. The data source was a written think aloud protocol on diagnosing a case of hyponatremia.

Result: Diagnostic performance for novices, intermediates, and experts was $0 \%, 80 \%, 83 \%$, respectively. At the students' level, $30 \%(2 / 6)$ had reduced knowledge in this clinical presentation. Seven out of ten residents demonstrated an abridged knowledge structure. From the seven internists, two expressed an abridged knowledge structure, three had a compiled/scheme knowledge structure, and two had scripted knowledge structures. Knowledge structure was statistically predictive of diagnostic success $(\beta=0.70, p<0.001)$

Conclusions: This study provides some insight into the knowledge structure and problem solving strategies at various levels of expertise. Medical education planning in Saudi Arabia should focus on enhancing expertise development and recognize the importance of a broad medical knowledge domain while for making the learning objectives explicit for learners.

Keywords: Knowledge structure; Problem solving; Diagnostic performance

\section{Introduction}

Health foundation starts as early as undergraduate medical education. In the Kingdom of Saudi Arabia (KSA) a dramatic expansion has occurred in the number of medical schools within a short period. The first medical school was established in 1967. Four other medical schools were established in the period from 1967 until 1996. Since the new millennium till today medical schools expanded dramatically in both the government and private sector to include 25 medical schools. This increase in medical schools is needed to cover the shortage of Saudi physicians in KSA [1]. On the other hand, maintaining a high quality standard of education with up-to date evidence based medical education is an obligation. The educational system at the medical schools has to be efficient and effective in developing experts in the medical field to meet the health needs and expectations of the Saudi community.

Graduates from these medical schools expand their expertise through deliberate practice and exposure to patients in the clinical setting. Studies of expertise highlighted some main features that distinguish experts from non-experts [2]. Experts have acquired more knowledge in their domain. They have organized hierarchal structured knowledge and can better identify key-features that facilitate differentiating one cause of a disease from another. In medical education the basic elements defining expertise are knowledge, knowledge structure and processes of clinical reasoning [3].

As the proficiency levels of learner's advances from a novice to an intermediate to an expert, a progression of knowledge structure and a change of the clinical problem solving methods utilized occur [4]. The knowledge structure evolves with deliberate practice and experience through the following stages: reduced, dispersed, elaborated causal, scheme, and scripts (Figure1).

In the stage of reduced knowledge, the learner has decreased knowledge of diseases and their manifestations. When knowledge of the diseases increases but with less developed manifestations of each disease, the learner is in a dispersed knowledge stage and ultimately uses hypothetical deductive reasoning (i.e., testing of one hypothesis at a time) as a clinical problem solving strategy. Further development and increase in knowledge of diseases and their manifestations with causal relationships characterizes the elaborated causal stage. In this stage of an intermediate proficiency level, hypothetical deductive reasoning is used and better diagnostic performance is expected in comparison to the reduced stage.

The evolution of knowledge and knowledge structure into a hierarchal organized scheme with limited number of key features to discriminate each category is the scheme stage. This level of expertise is advanced and involves forward scheme inductive reasoning. It is

*Corresponding author: FM Munshi, Department of Medical Education and Educational Technology, Faculty of Medicine, King Fahad Medical City at King Saud Bin Abdulaziz University for Health Sciences, Riyadh, Saudi Arabia, Tel: 4033974419; E-mail: drmunshi@gmail.com

Received September 03, 2013; Accepted November 21, 2013; Published November 29, 2013

Citation: Munshi FM, AlJarallah BM, Harasym PH (2013) Problem Solving Strategy and Diagnostic Performance at Three Expertise Levels: Does the Problem Solving Approach Matter? J Biosafety Health Educ 1: 110. doi:10.4172/2332-0893.1000110

Copyright: (c) 2013 Munshi FM, et al. This is an open-access article distributed under the terms of the Creative Commons Attribution License, which permits unrestricted use, distribution, and reproduction in any medium, provided the original author and source are credited. 


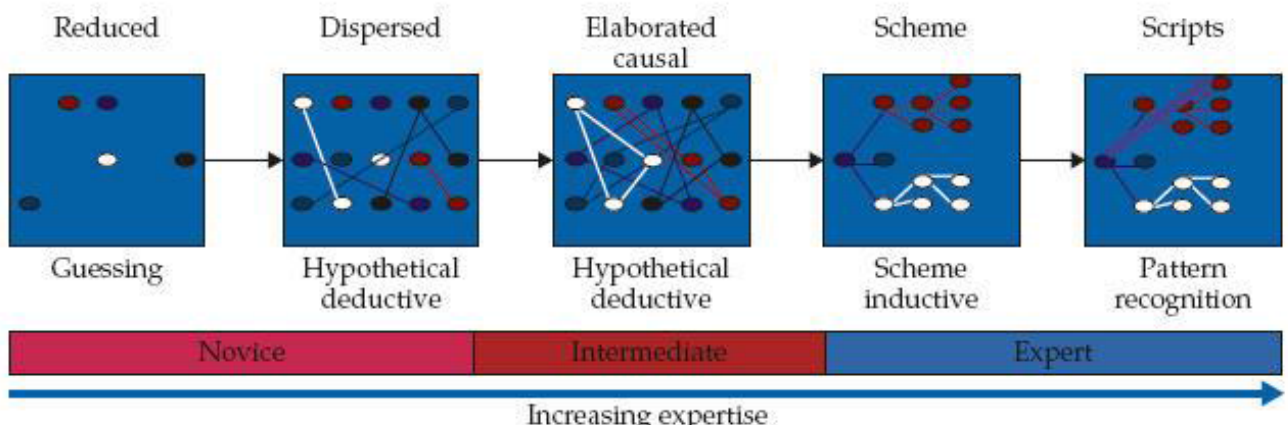

Figure 1: Evolution of knowledge structure from novice to intermediate to expert [4].

associated with better diagnostic performance. With extended exposure to patients, an expert further establishes key features of diseases in a scheme, which enable recognition of diseases using pattern recognition.

The knowledge structure and organization are eminent in expertise development. The study of knowledge structure has two methods, direct and indirect [5]. Indirect methods often employ statistical procedures to determine the knowledge and tend to give insight into the relation between facts. Examples are multidimensional scaling, hierarchal clustering and concept sorting. Direct methods assume that knowledge can be verbalized by the subject and appropriately classified by trained individuals to give insight to the knowledge and knowledge structure. Examples include protocol analysis, interview and questionnaire. The objective of this study was to use the direct method of inquiry to explore the knowledge structure, problem solving strategy, and diagnostic performance at three levels of expertise (novice, intermediate and expert).

\section{Methods}

\section{Research design, institutions and subjects}

This was a cross sectional study. Novice level participants included six medical students in their fifth year of the MD program that had completed their rotation in the medical department at King Fahad Medical City (KFMC), Faculty of Medicine in Riyadh, Saudi Arabia. The curriculum at KFMC is a six year PBL curriculum in which the first year is a premedical year. A year consists of eight months and contains eight to ten blocks of instruction/learning opportunities. In a block, one problem/clinical case is discussed on a weekly basis.

Intermediate expertise level participants were 10 internal medicine residents at various training years at KFMC, Medical Department. Expert participants were internists in Department of Medicine at Qassim University. The seven internists whom volunteered to be part of this study had at least 3 years of clinical experience. In brief, this was a convenience sample reflective of three expertise levels with twentythree participants.

\section{Clinical case}

The data source was a written think aloud protocol, with no time constraint, on diagnosing a written clinical problem of hyponatremia (Appendix 1). Participants were given a written clinical vignette with eight diseases to choose from. Then, they were asked to describe the thinking process they went through to reach their chosen diagnosis.

The authors FMM and BMA independently rated the knowledge structure and problem solving approach used by each participant. Each author was blind to the level of expertise. Discrepancies in decisions were resolved through discussion. The direction of problem solving and number of concepts related to hyponatremia were the main factors used in categorizing the written think aloud protocols.

The linear relationship between the independent variables, (i.e., level of expertise, knowledge structure, and problem solving approach) were examined with the dependant variable diagnostic performance, using Pearson correlation coefficient. Linear regression analysis was also computed to determine the nature and strength of relationships among the variables of interest.

\section{Results}

The knowledge structure and problem solving approach used by each participant were independently rated by the authors (F.M.M and B.M.A.). The inter-rater agreement between authors F.M.M and B.M.A. was $90 \%$. Discussion enabled $100 \%$ agreement in classification of knowledge structure and reasoning strategy used.

At the novice level, the fifth year medical students in this study (6/6) chose the wrong diagnosis (Figure 2). Thirty-three percent $(2 / 6)$ of the students had reduced knowledge in this clinical presentation (Figure 3 ) and tried to guess the diagnosis (Figure 4). One of the students expressed his thoughts as the following:

"I thought about the most common cause of hyponatremia, I think it is related to water intake which should be increased. I think it also has something to do with increased urine osmolality, but I'm not sure".

Seventy percent $(4 / 6)$ of the students had dispersed knowledge and utilized a hypothetical deductive approach for problem solving and got the wrong diagnosis.

At the intermediate level of expertise, $80 \%(8 / 10)$ of the residents got the correct answer (Figure 2). These residents demonstrated an abridged knowledge structure with a hypothetical deductive approach except for one resident (Figures 3 and 4). This resident expressed a compiled knowledge structure and utilized a scheme inductive method in problem solving. He described the thinking process as follows:

"This young female has a truly low hypotonic hyponatremia and very low urine osmolality, this happens in two conditions only, my diagnosis is primary polydipsia".

At the level of the expert internists, one internist out of seven (14\%) got the wrong diagnosis (Figure 2). He used a hypothetical deductive problem solving method with abridged knowledge. Of the seven internists, two (29\%) expressed an abridged knowledge structure and 


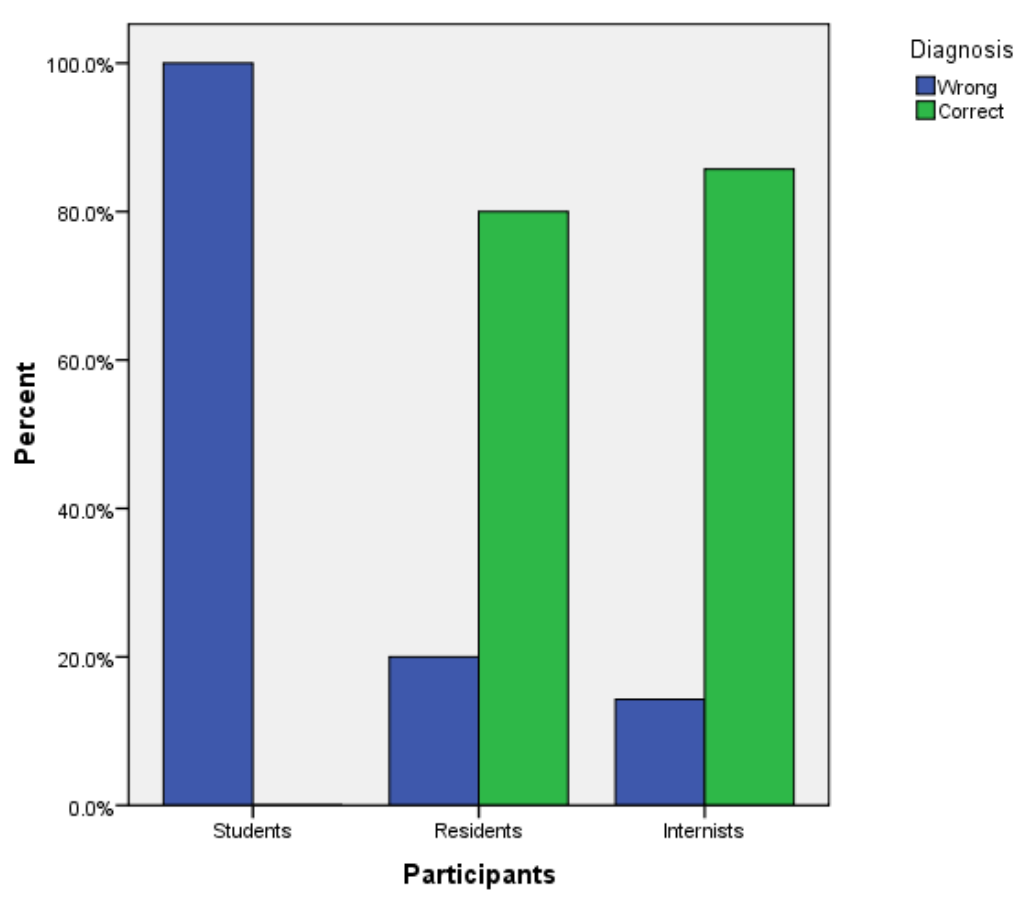

Figure 2: A graph representing the Diagnostic Performance for Students, Residents and Internists.

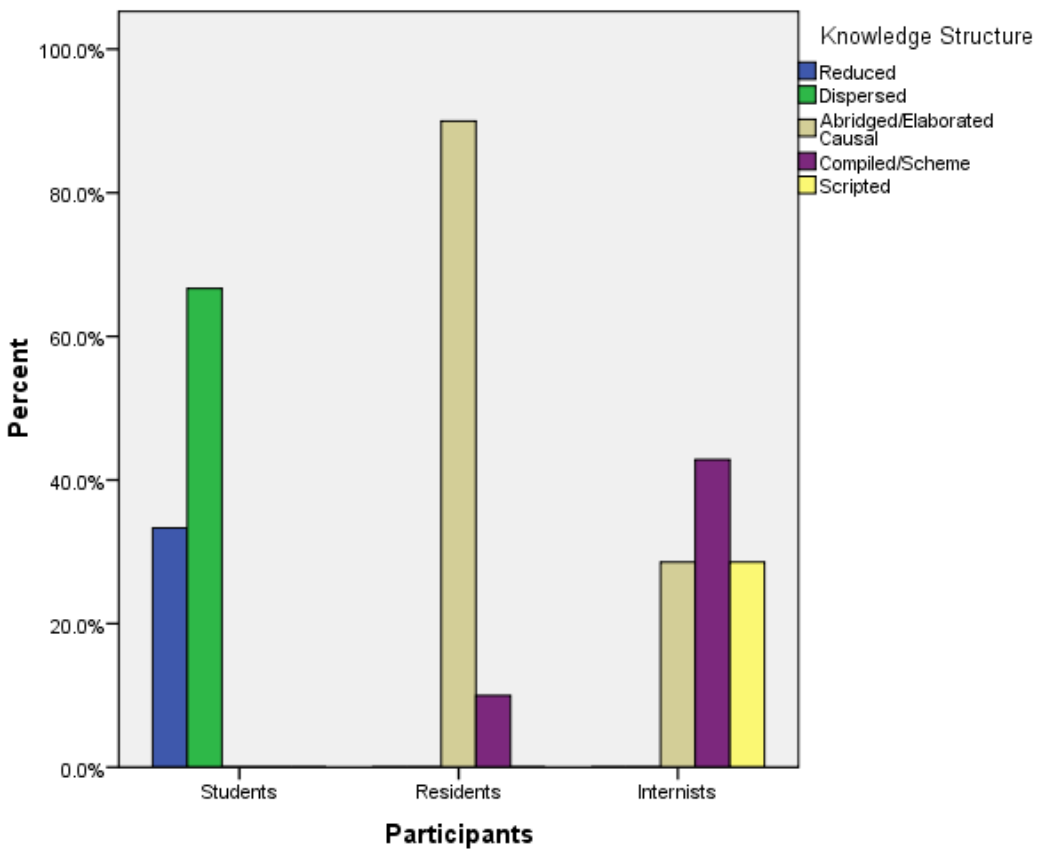

Figure 3: A graph representing the Knowledge Structure used by Students, Residents and Internists to diagnose a clinical presentation of hyponatremia.

used a hypothetical deductive approach (Figures 3 and 4). A compiled/ scheme knowledge structure was used by three (43\%) internists with a scheme inductive approach. Two (29\%) internists had scripted knowledge structures and utilized a pattern recognition method. One of them expressed his thinking process as:

"Primary polydipsia because both serum and urine osmolality are low with loss of sodium"
Level of expertise, knowledge structure and problem solving approach significantly $(\mathrm{p}<0.05)$ correlated with diagnostic performance, $0.64,0.70,0.49$ respectively. Inclusion of the three independent variables in a stepwise regression analysis as predictors of diagnostic performance revealed that only knowledge structure was statistically predictive of diagnostic performance $(\beta=0.70, p<0.001)$, which explained $50 \%$ variance. 


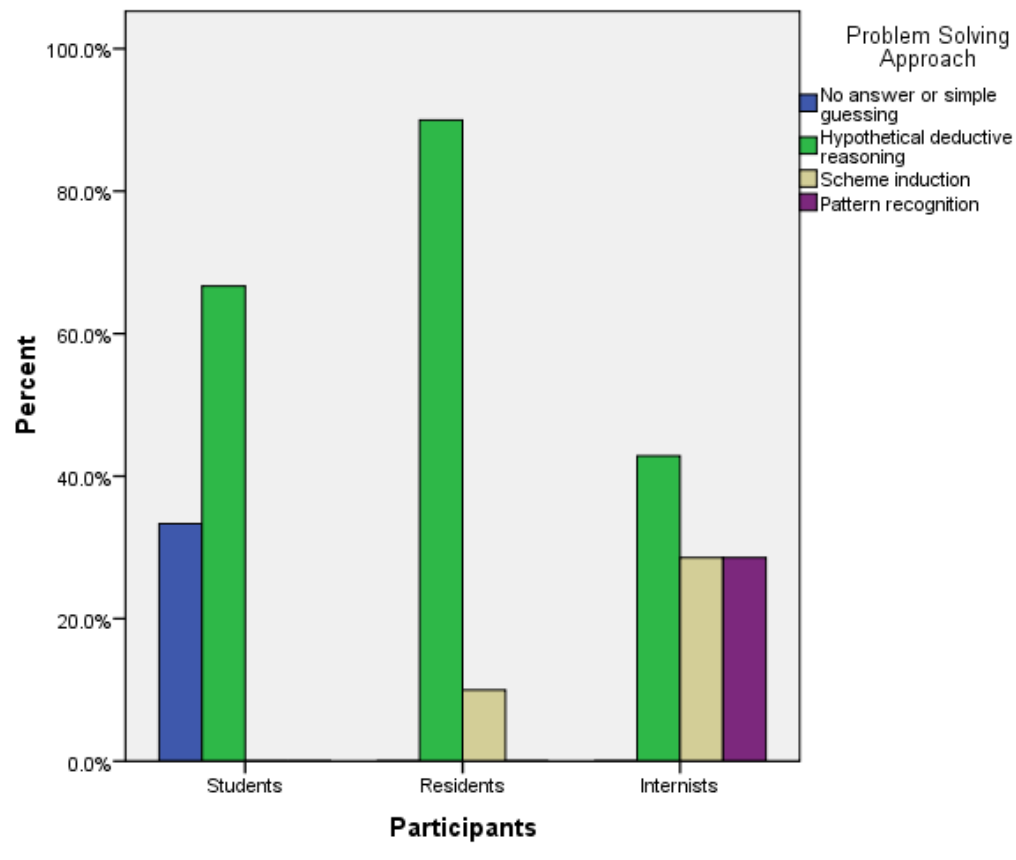

Figure 4: A graph representing the Problem Solving Approach used by Students, Residents and Internists to diagnose a clinical presentation of hyponatremia

\section{Discussion and Conclusion}

This study used a written think aloud protocol analysis to explore the knowledge structure, problem solving approach and the diagnostic accuracy at three expertise levels. This study supports the findings of similar studies of clinical reasoning strategies and diagnostic accuracy $[5,6]$. Participants using a scheme inductive or a pattern recognition diagnostic problem solving strategy had better diagnostic accuracy.

The medical school at KFMC is one of the medical schools in KSA that adopted a hybrid PBL undergraduate curriculum. In a PBL curriculum, students are encouraged to list as many hypotheses as possible during the problem solving session. It is not a surprise that all the fifth year students in this study got the diagnosis wrong. Problem solving is not a generic process in which knowledge attained in a certain clinical problem can be applied to other problems [7].

It appears from the analysis of the students' descriptions that they have reduced or dispersed knowledge in this clinical presentation of hyponatremia. Probably, the fifth year students in this study did not come across this clinical disease. This case specificity phenomena has led the University of Calgary to develop the Clinical Presentation Model [7] .

In the process of developing the clinical presentation curriculum, coverage of the knowledge domain was a key factor, and an incomplete set of problems was considered a drawback. Therefore, in the clinical presentation curriculum students are exposed to 3000-3500 diseases throughout the medical degree program. While in a problem based curriculum, students' exposure is limited to $150-450$ diseases [8].

At the level of the residents, final year resident used a scheme induction strategy to diagnose the clinical case. The other residents were first and second year residents. Two of the first year residents got the diagnosis wrong. This should draw attention to their knowledge base in this field.
The clinical presentation used in this study is perceived as a prototypical case at the level of medical students. One of the factors significantly associated with diagnostic performance is the extended experience and advanced level of expertise [6]. Five of the internists had an organized knowledge structure ranging from complied to script. Experience and deliberate practice leads to this level of expertise but in general it takes around ten years to develop an expert in a domain. Clinical presentation curriculum provides the students with problems driven from cognitive task analysis similar to that used by expert physicians.

The expert schemes, which usually take years to develop in practice, are presented to students to guide their learning and knowledge organization [7]. Interesting to note, advanced knowledge structure was the main statistically predictive factor of diagnostic success.

This study sheds insight into the knowledge structure and problem solving strategies at various levels of expertise using a convenience sample. Medical education planning in Saudi Arabia should consider two implications. First, educational planners should recognize the importance of a broad medical knowledge domain while making the learning limits explicit for learners. Second, planners should focus on enhancing expertise by teaching students advanced clinical problem solving skills and assisting them to develop an organized knowledge structure. This can be achieved by using expert developed schemes [9].

Nevertheless, there are few limitations of this study. Firstly, only one clinical presentation was used to study the knowledge structure and problem solving strategy. Although, this problem was conceived as a prototypical problem, other clinical presentations could be explored in the future. Secondly, the number of participants in this study limits the generalizability of these findings.

The study demonstrates frequencies of knowledge structure, problem solving strategy and diagnostic accuracy at three expertise levels in Saudi Arabia. It also reveals the need for medical education 
Citation: Munshi FM, AlJarallah BM, Harasym PH (2013) Problem Solving Strategy and Diagnostic Performance at Three Expertise Levels: Does the Problem Solving Approach Matter? J Biosafety Health Educ 1: 110. doi:10.4172/2332-0893.1000110

planners to study and revise the current educational settings in depth and consider some of the implications described in this study.

\section{Reference}

1. Mufti M (2000) Healthcare Development Strategies in the Kingdom of Saudi Arabia. Springer

2. Ericsson KA, Charness N, Feltovich PJ, Hoffman RR (2006) The Cambridge Handbook of Expertise and Expert Performance (Cambridge Handbooks in Psychology). Cambridge University Press, UK.

3. Schmidt HG, Norman GR, Boshuizen HP (1990) A cognitive perspective on medical expertise: theory and implication. Acad Med 65: 611-621.

4. Harasym PH, Tsai TC, Hemmati P (2008) Current Trends in Developing Medical Students' Critical Thinking Abilities. Kaohsiung J Med Sci 24: 341-355.
5. McLaughlin K, Coderre S, Mortis G, Mandin H (2007) Expert-Type Knowledge Structure in Medical Students is Associated with Increased Odds of Diagnostic Success. Teach Learn Med 19: 35-41.

6. Coderre S, Mandin H, Harasym PH, Fick GH (2003) Diagnostic reasoning strategies and diagnostic success. Med Educ 37: 695-703.

7. Kirschner PA, Sweller J, Clark R E (2006) Why Minimal Guidance During Instruction Does Not Work: An Analysis of the Failure of Constructivist Discovery, Problem-Based, Experiential, and Inquiry-Based Teaching. Educ Psychol 41: 75-86.

8. Papa FJ, Harasym PH (1999) Medical curriculum reform in North America, 1765 to the present: a cognitive science perspective. Acad Med 74: 154-164.

9. Mandin H, Jones A, Woloschuk W, Harasym P (1997) Helping students learn to think like experts when solving clinical problems. Acad Med 72: 173-179. 